\title{
Erratum to: Stickler syndrome caused by COL2A1 mutations: genotype-phenotype correlation in a series of 100 patients
}

Kristien P Hoornaert, Inge Vereecke, Chantal Dewinter, Thomas Rosenberg, Frits A Beemer, Jules G Leroy, Laila Bendix, Erik Björck, Maryse Bonduelle, Odile Boute, Valerie Cormier-Daire, Christine De Die-Smulders, Anne Dieux-Coeslier, Hélène Dollfus, Mariet Elting, Andrew Green, Veronica I Guerci, Raoul CM Hennekam, Yvonne Hilhorts-Hofstee, Muriel Holder, Carel Hoyng, Kristi J Jones, Dragana Josifova, Ilkka Kaitila, Suzanne Kjaergaard, Yolande H Kroes, Kristina Lagerstedt, Melissa Lees, Martine LeMerrer, Cinzia Magnani, Carlo Marcelis, Loreto Martorell, Michèle Mathieu, Meriel McEntagart, Angela Mendicino, Jenny Morton, Gabrielli Orazio, Véronique Paquis, Orit Reish, Kalle OJ Simola, Sarah F Smithson, Karen I Temple, Elisabeth Van Aken, Yolande Van Bever, Jenneke van den Ende, Johanna M Van Hagen, Leopoldo Zelante, Riina Zordania, Anne De Paepe, Bart P Leroy, Marc De Buyzere, Paul J Coucke and Geert R Mortier

European Journal of Human Genetics (2010) 18, 881; doi:10.1038/ejhg.2010.37

Correction to: European Journal of Human Genetics (2010) 18, 872-880; doi:10.1038/ejhg.2010.23; published online 24 February 2010

Affiliation 1 appears incorrect in the paper. The correct affiliation should be Center for Medical Genetics, Ghent University Hospital, Gent, Belgium.
Geert $\mathrm{R}$ Mortier has two different affiliations: affiliation 1 and Department of Medical Genetics, Antwerp University Hospital, Prins Boudewijnlaan 43, B-2650 Edegem, Belgium.

The typesetters would like to apologise for this mistake. 